\title{
The Impact of Protein Corona in Nanoflare-based Biomolecular Detection and Quantification
}

Hao Wang, ${ }^{a}$ Kholud Dardir, ${ }^{a}$ K.-B. Lee, ${ }^{b}$ and Laura Fabris ${ }^{a *}$

a. Department of Materials Science and Engineering, Rutgers university, 607 Taylor Road, Piscataway, NJ, 08854 USA

b. Department of Chemistry and Chemical Biology, Rutgers university, 607 Taylor Road, Piscataway, NJ, 08854 USA

*corresponding author email: Ifabris@soe.rutgers.edu

\section{Supporting Information}

\section{Table of Contents}

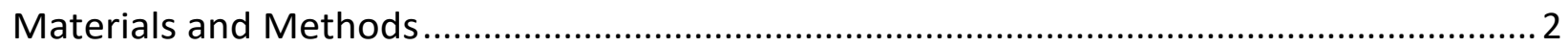

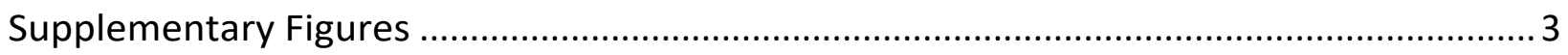

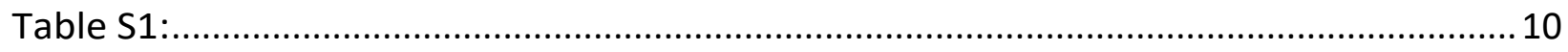

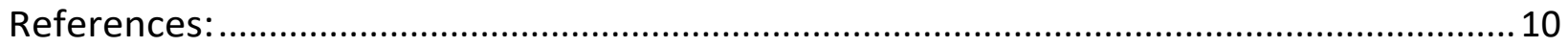




\section{Materials and Methods}

Materials: Tetrachloroauric(III) acid trihydrate $\left(\mathrm{HAuCl}_{4} \cdot 3 \mathrm{H}_{2} \mathrm{O}\right)$, trisodium citrate dihydrate $\left(\mathrm{C}_{6} \mathrm{H}_{5} \mathrm{O}_{7} \mathrm{Na} \cdot 2 \mathrm{H}_{2} \mathrm{O}\right)$ and $\mathrm{L}(+)$-ascorbic acid $\left(\mathrm{C}_{6} \mathrm{H}_{8} \mathrm{O}_{6} ; \geq 99 \%\right)$, hydrochloric acid $(\mathrm{HCl} ; 36.5 \%$ to $38.0 \%)$ were purchased from Fischer Scientific. Silver nitrate $\left(\mathrm{AgNO}_{3} ; \geq 99.995 \%\right)$, potassium cyanide (KCN; $\geq 96.0 \%)$, sodium chloride $(\mathrm{NaCl} ; \geq 99 \%)$, potassium phosphate monobasic $\left(\mathrm{KH}_{2} \mathrm{PO}_{4} ; \geq 99 \%\right)$, potassium phosphate dibasic $\left(\mathrm{K}_{2} \mathrm{HPO}_{4} ; \geq 98 \%\right)$, tris(2-carboxyethyl)phosphine hydrochloride $\left(\mathrm{C}_{9} \mathrm{H}_{15} \mathrm{O}_{6} \mathrm{P} \cdot \mathrm{HCl}\right.$; TCEP), human serum albumin (lyophilized powder, $\geq 96 \%$ ), bovine serum albumin (lyophilized powder; $\geq 96 \%$ ), fibrinogen (lyophilized powder, $\geq 80 \%$ ) were all purchased from Sigma Aldrich. DNase I, RNase-free $(1 \mathrm{U} / \mu \mathrm{L})$ was purchased from Thermo-Fisher Scientific. The DNA sequences (Table 1) used in this study were all purchased from Integrated DNA Technologies. All these chemicals were used without further purification. Ultrapure water $(18.2 \mathrm{M} \Omega \cdot \mathrm{cm})$ was used in preparing all aqueous solutions. All glassware was cleaned by aqua-regia and thoroughly rinsed by ultrapure water before nanoparticle synthesis or storage.

Nanoparticle synthesis: Typically, gold nanoparticle spheres ( $15 \mathrm{~nm})$ were prepared by adding 3.75 $\mathrm{mL}$ of $1 \%$ trisodium citrate solution into $62.5 \mathrm{~mL}$ of HAuCl 4 solution $(0.4 \mathrm{mM})$ heated to rolling boil under gentle stirring. Within 10 minutes, the colour of the solution changed from pale yellow to colourless and eventually burgundy. The mixture was then allowed to cool down to room temperature while stirring. The prepared gold seeds were then purified by three rounds of centrifugation at $8500 \mathrm{rpm} \times 30 \mathrm{~min}$. Then the purified gold seeds were resuspended in ultra-pure water and stored at $4{ }^{\circ} \mathrm{C}$ until further use. The surfactant-free gold nanostars were synthesized by following a seed-mediated growth protocol previously reported by the Vo-Dinh group ${ }^{1}$. Briefly, $100 \mu \mathrm{L}$ of $1 \mathrm{~N} \mathrm{HCl}$ and $62.5 \mu \mathrm{L}$ of gold seeds (O.D. $=\sim 2.81$ ) were added into $25 \mathrm{~mL} \mathrm{HAuCl}_{4}$ solution $(1 \mathrm{mM})$ and mixed thoroughly through gentle stirring. Then, very quickly, $1 \mathrm{~mL}$ of $\mathrm{AgNO}_{3}(3 \mathrm{mM})$ and $500 \mu \mathrm{L}$ of $\mathrm{L}(+)$-ascorbic acid $(100 \mathrm{mM})$ were simultaneously added to the above mixture. The solution immediately turned greenish-black. After 7 min of stirring, the reaction was halted by centrifugation at $4000 \mathrm{rpm} \times 15 \mathrm{~min}$ for three rounds. The final products of nanostars ( 50 nm) were then resuspended with ultra-pure water and stored under $4{ }^{\circ} \mathrm{C}$ until further use. UV-Vis measurements were conducted with a typical Ocean Optics set up or Nanodrop One UV-Vis spectrophotometer with a plastic cuvette and $\sim 1 \mathrm{~mL}$ sample. TEM images were obtained by JEOL 1200EX electron microscope equipped with an AMT-XR41 digital camera.

Nanoflares functionalization: Flare sequences were first mixed with corresponding sensing sequences (1.2:1) in phosphate buffered saline (PBS: $137 \mathrm{mM} \mathrm{NaCl}, 10 \mathrm{mM}$ Phosphate, $2.7 \mathrm{mM}$ $\mathrm{KCl}, \mathrm{pH} 7.4$ ), heated to $75{ }^{\circ} \mathrm{C}$ and maintained for $10 \mathrm{~min}$, then slowly cooled to room temperature and stored in the dark for at least 12 hours to allow complete hybridization. These flare duplexes were then treated by TCEP for 3 hours to break the dithiol bonds and activate the formed duplex strands. Then these reduced duplex strands were mixed with either gold nanoparticle spheres or gold nanostars and incubated in the dark overnight. Then $10 \%$ SDS was added into the mixture to achieve a final concentration of SDS $0.1 \%$ and phosphate buffer $(0.1 \mathrm{M})$ added to achieve a final concentration of $0.01 \mathrm{M}$. NaCl $(3 \mathrm{M})$ was then slowly added into the mixture to increase the concentration of $\mathrm{NaCl}$ to $0.3 \mathrm{M}$ in a $6 \sim 8$ hour period. Then the solution containing nanoflares was centrifuged $(12,000 \mathrm{rpm}, 20 \mathrm{~min})$ and resuspended in PBS (1x) $3 \sim 4$ times and stored at $4{ }^{\circ} \mathrm{C}$ before further use.

Protein corona formation: Prepared nanoflares were typically incubated with solutions of human serum albumin (HSA), bovine serum albumin (BSA) or fibrinogen (FN) in PBS at a biologically relevant concentration (0.50 wt\%) or at elevating concentrations (0.10 wt\% to $2.50 \mathrm{wt} \%)$, overnight at $37.5^{\circ} \mathrm{C}$ before further measurements.

Dynamic light scattering (DLS) measurement: The sizes of the as-synthesized nanoparticles, nanoflares, and corona encapsulated nanoflares were measured on Zetasizer Ultra and analyzed by ZS Xplorer (Malvern). The surface charges of these different nanoparticles were measured on the same instrument with a surface zeta potential cell purchased from Malvern. All measurements were conducted by triplicates for statistical significance. 
Fluorescence measurement: For fluorescence spectral response measurements, complementary target (or random) sequences were added into $\sim 1 \mathrm{~mL}$ of nanoflares sample solution and thoroughly mixed. Then, fluorescence spectra were recorded at various time points (e.g. 2, 5, 10, $20,30,40,50,60 \mathrm{~min}$.) until the fluorescence signal completely plateaued. These measurements were typically implemented on a single-cell Cary Eclipse fluorimeter. For concentration dependence studies, fluorescence kinetics was measured for nanoflares treated with different concentrations of the target sequence (e.g. $0,5,10,25,50,100,200 \mathrm{nM}$, etc.) by continuously monitoring the fluorescence signal at the proper emission wavelength. Probing the sequence specificity of the nanoflares, the fluorescence signal was monitored continuously for $\sim 60 \mathrm{~min}$ after the addition of the same concentration of fully complementary, 1 mismatched and 2 mismatched targets, as well as random sequences (see Table S1 for details). For nuclease catalysed hydrolysis of the as-synthesized nanoflares and corona-functionalized nanoflares, fluorescence kinetics were measured for different nanoflares (concentration kept the same as determined by the absorption intensity in UV-Vis) treated with DNase I ( $2 \mathrm{U} / \mathrm{L}$ ) by continuously monitoring for at least 60 minutes the fluorescence signal of the digested flare strands with 6-FAM labels. These measurements were implemented with a microplate reader (Molecular Devices, M2) for its multi-channel and highthroughput capabilities. The fluorescence of 6 -FAM was excited at $488 / 485 \mathrm{~nm}$ and measured at $518 / 525 \mathrm{~nm}$. The fluorescence of Cy5 was excited at $645 / 630 \mathrm{~nm}$ and measured at $668 \mathrm{~nm}$. Quantification of the surface loading of flares on the NPs was carried out using an established procedure ${ }^{2}$, using KCN $(0.1 \mathrm{M})$ for gold dissolution.

Raman measurement: Raman measurements were performed for nanoflares before and after exposure to target sequences on a portable Raman system (Hamamatsu photonics, C12710 module, $\lambda=785 \mathrm{~nm}$, maximum laser power $50 \mathrm{~mW}$ ). The Raman signals were acquired by focusing the laser on the nanoflares solution contained in a suitable well with a $10 \mathrm{~s}$ acquisition time and 3 repetitions.

\section{Supplementary Figures}

a)

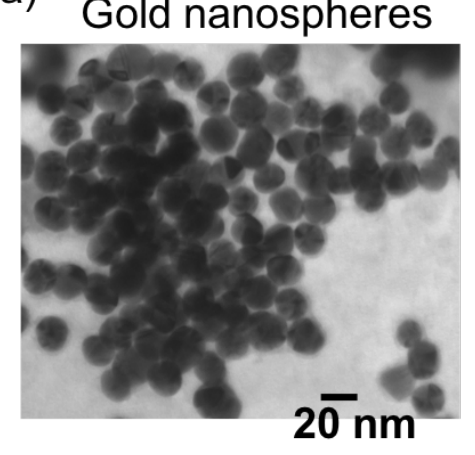

b)

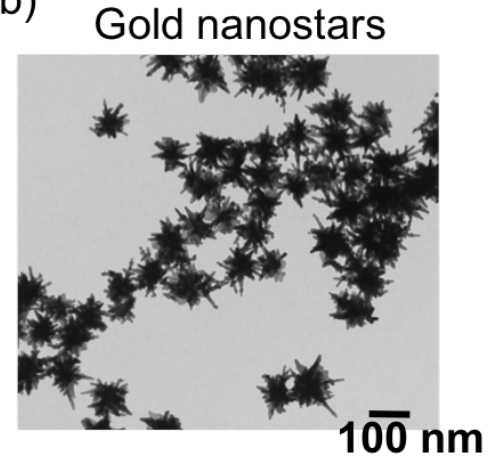

c)

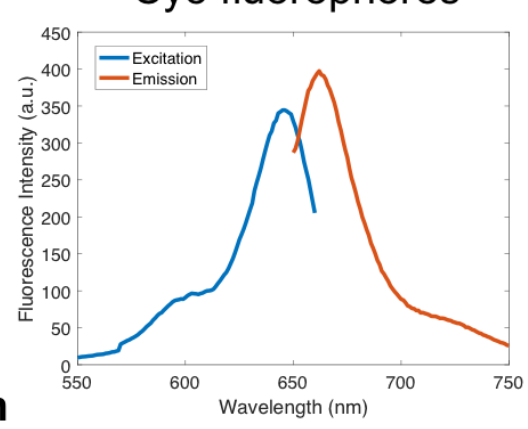

Figure S1: TEM images of as-synthesized a) nanospheres and b) nanostars. c) Excitation and emission profiles of the Cy5 fluorophores. 
a)

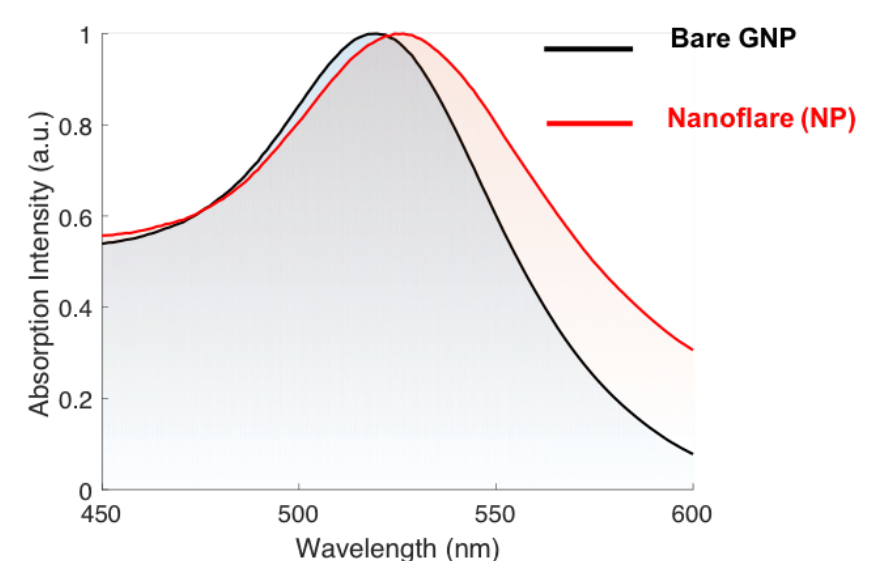

b)

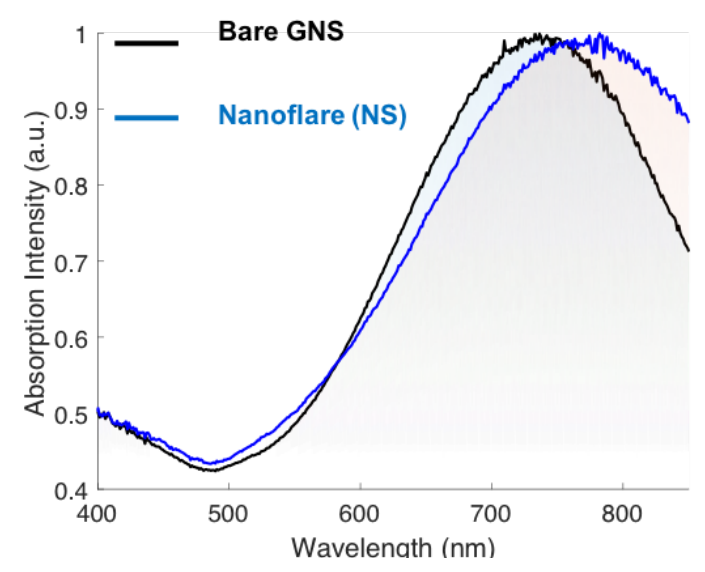

Figure S2: UV-Vis absorption profiles of a) unfunctionalized GNP vs. cy5-flr-GNP (peak shift from $520 \mathrm{~nm}$ to $524 \mathrm{~nm}$ ) and b) unfunctionalized GNS vs. cy5-flr-GNS (744 nm to $775 \mathrm{~nm}$ ).

a)

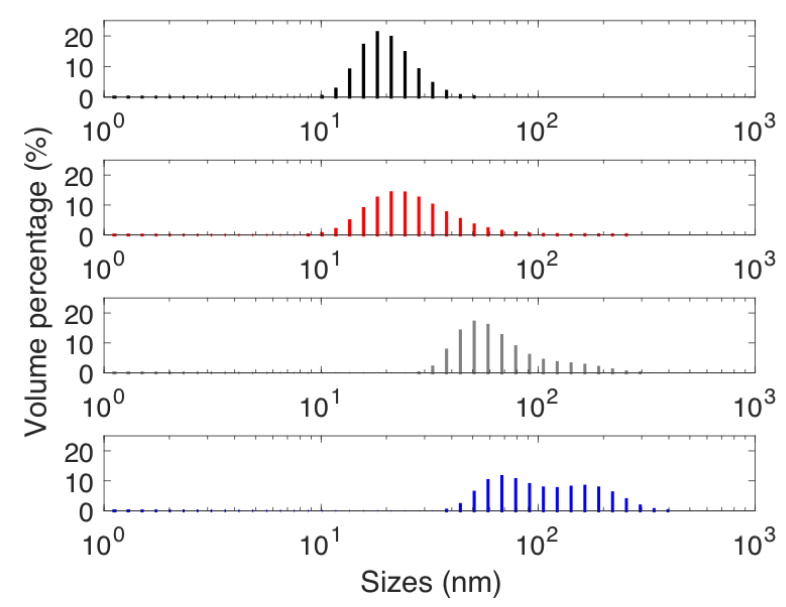

b)

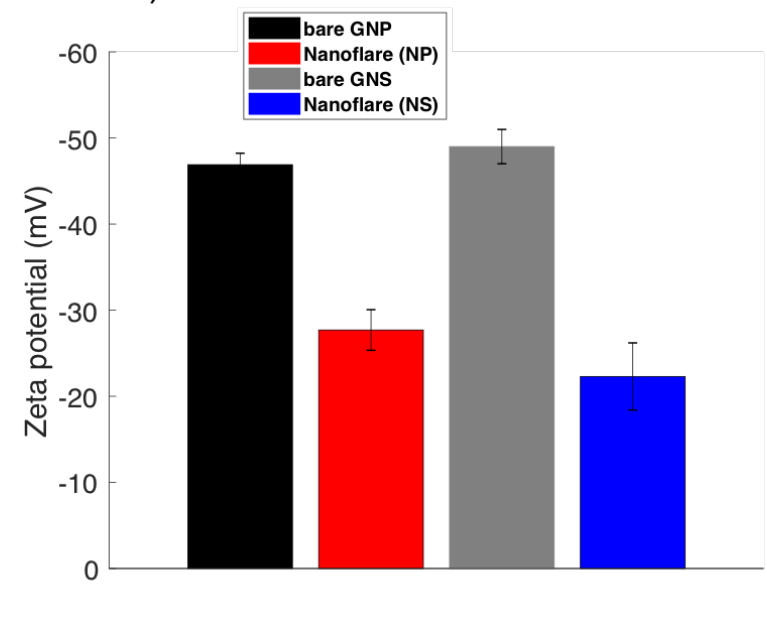

Figure S3: a) Changes in nanoparticle hydrodynamic size before and after DNA functionalization to the NP or NS surface; b) Changes in zeta potential of the NP or NS before and after flare functionalization. 

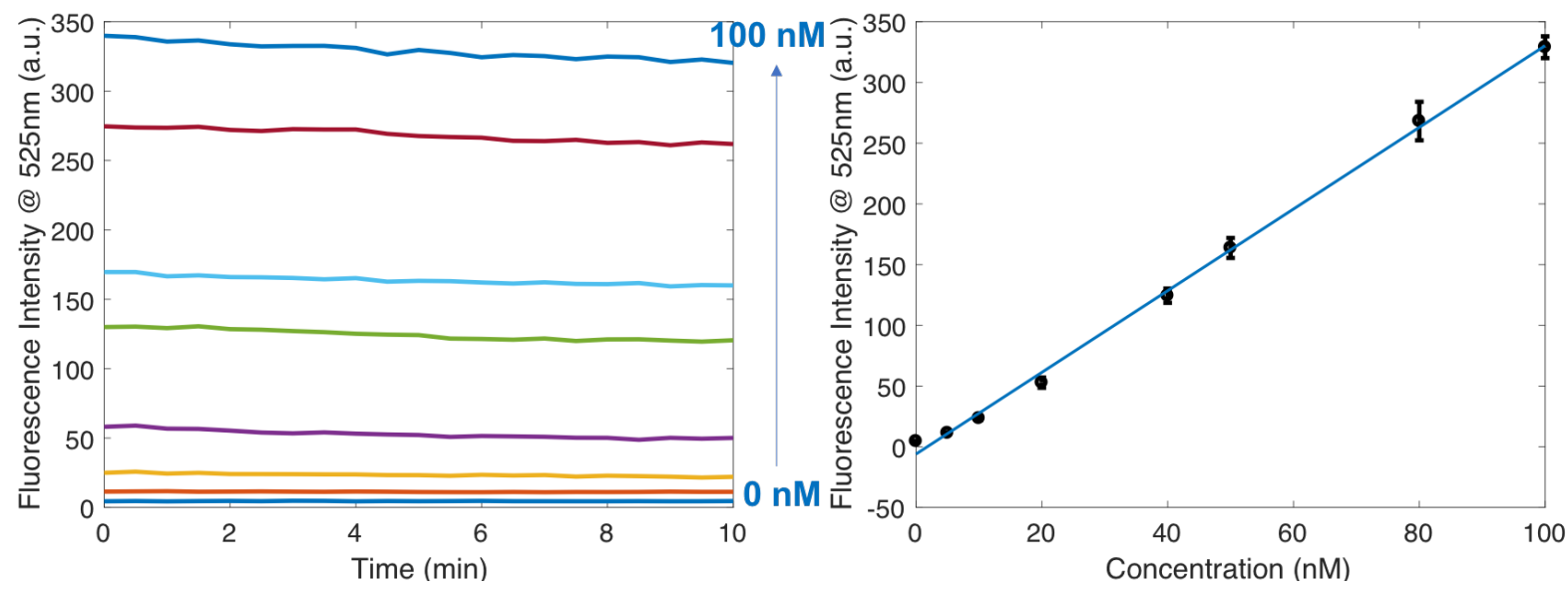

Figure S4: a) Measured fluorescence intensities of varying concentrations of 6-FAM labeled flare strands; b) Calibration curve built to calculate the surface loading of flares on the nanoparticle surfaces. A linear curve can be fitted: $y=3.364 * x-6.062\left(R^{2}=0.9977\right)$.

For a typical batch of NP-based nanoflares (OD $=0.622)$, after dissolution by $\operatorname{KCN}(0.1 \mathrm{M})$, fluorescence signal of 183 \pm 11 was obtained @ 525 nm emission. Based on the calibration curve in Fig. S4b, we can roughly estimate the flares loaded on the nanoparticle surface are $56.2 \pm 5.1 \mathrm{nM}$ while the nanoparticle concentration $\sim 2.3 \mathrm{nM}\left(\varepsilon=2.7 \times 10^{8} \mathrm{~cm}^{-1} \mathrm{M}^{-1}\right)$. For each individual nanoparticle, approximately $25 \pm 2$ flares are present on the surface.

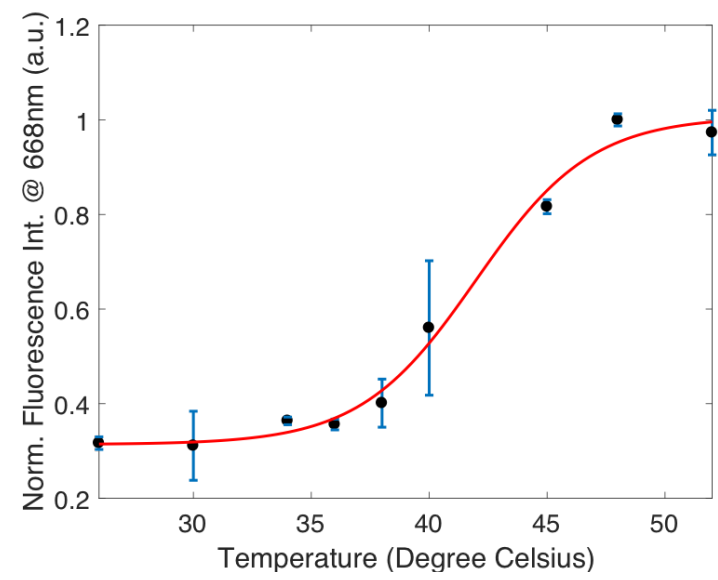

GTCCGACTACCAGACAGATTGATCCAATGT 6 -SH /5Cy5/TCATTGGATCAATCT

Calculated Tm for the flare strands: $\sim 36.6 \mathrm{deg}$

Measured Tm for nanoflare: $\sim 42.0 \mathrm{deg}$

Figure S5: Melting curve for the nanoflares (Cy5-flare-GNP) measured by monitoring the fluorescence signal changes while ramping the temperature from 25 to $52{ }^{\circ} \mathrm{C}$. The measured $\mathrm{T}_{\mathrm{m}}$ was measured to be $\sim 42{ }^{\circ} \mathrm{C}$, slightly higher than the calculated value corresponding to its molecular counterpart. ${ }^{3}$ 


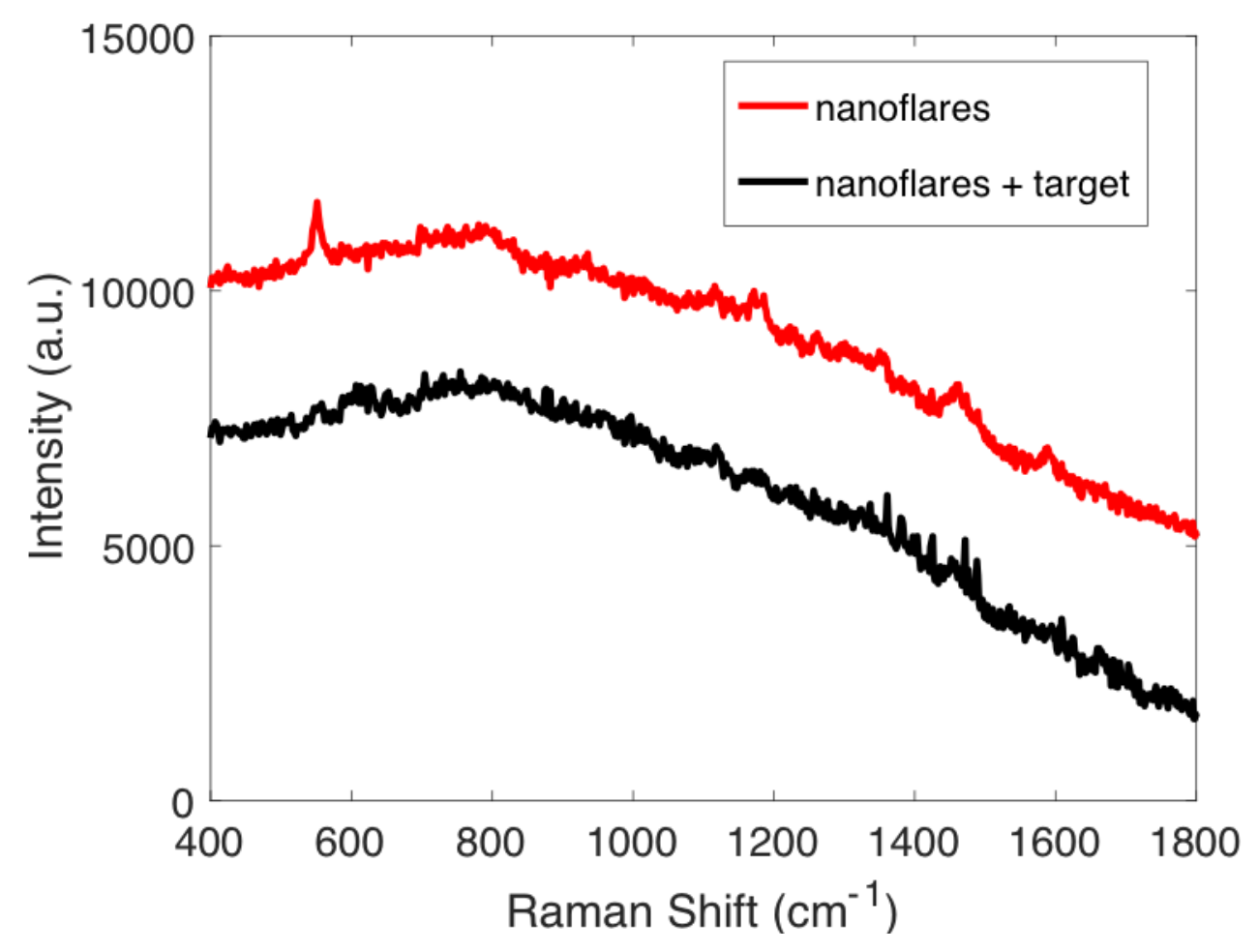

Figure S6: Surface enhanced Raman spectra obtained from NS-based nanoflares functionalized with Cy5-labeled flare sequences before and after hybridization with complementary targets. Raman peaks at $\sim 551 \mathrm{~cm}^{-1}, 1186 \mathrm{~cm}^{-1}, 1462 \mathrm{~cm}^{-1}, 1589 \mathrm{~cm}^{-1}$ can be assigned to Cy5 fluorophore. ${ }^{4}$
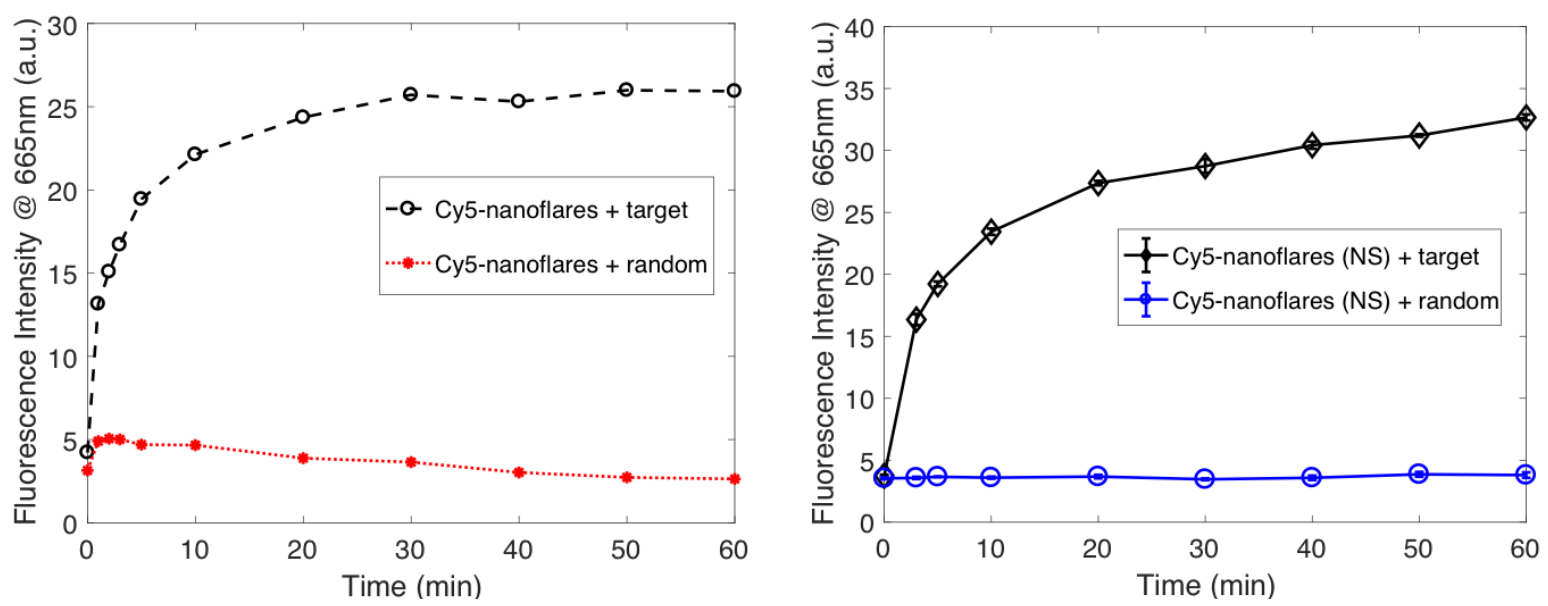

Figure S7: Fluorescence kinetics measured at $665 \mathrm{~nm}$ for $\mathrm{Cy} 5$ emission obtained from a) NP-based nanoflares interacting with either target or random sequences; b) NS-based nanoflares interacting with either target or random sequences. 
a)

b)

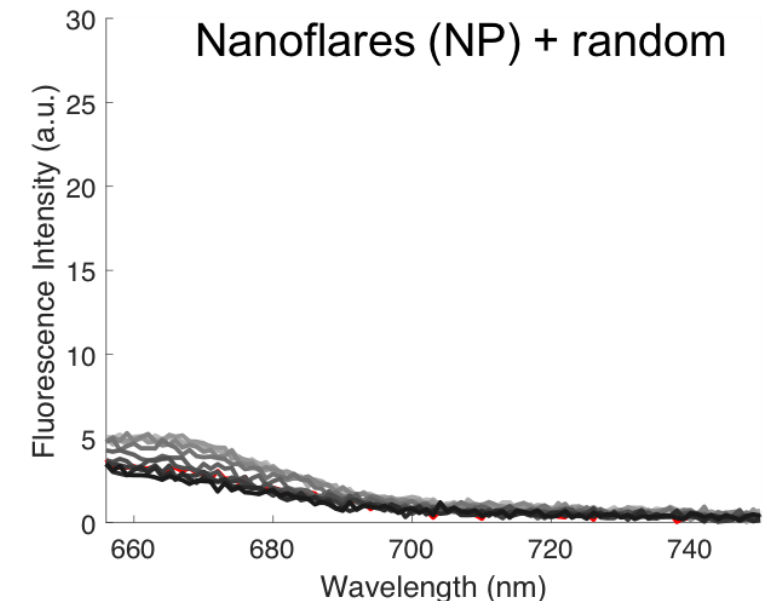

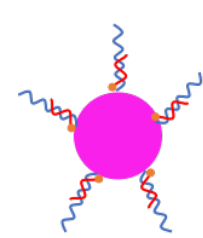
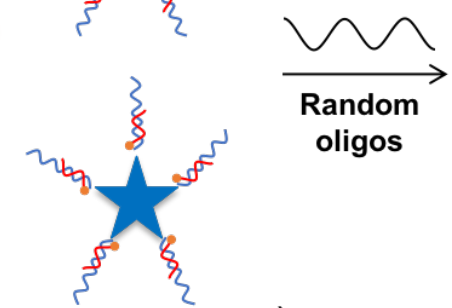

Random

oligos
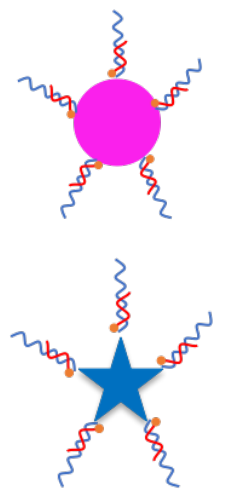

c)

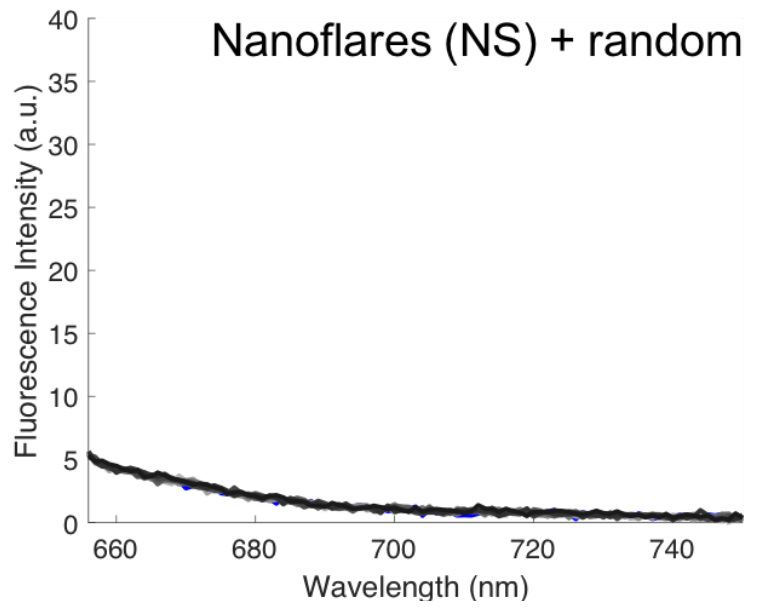

Figure S8: Fluorescence spectral changes obtained from a) NP-based nanoflares when exposed to scrambled random sequences; b) NS-based nanoflares when exposed to scrambled random sequences. 

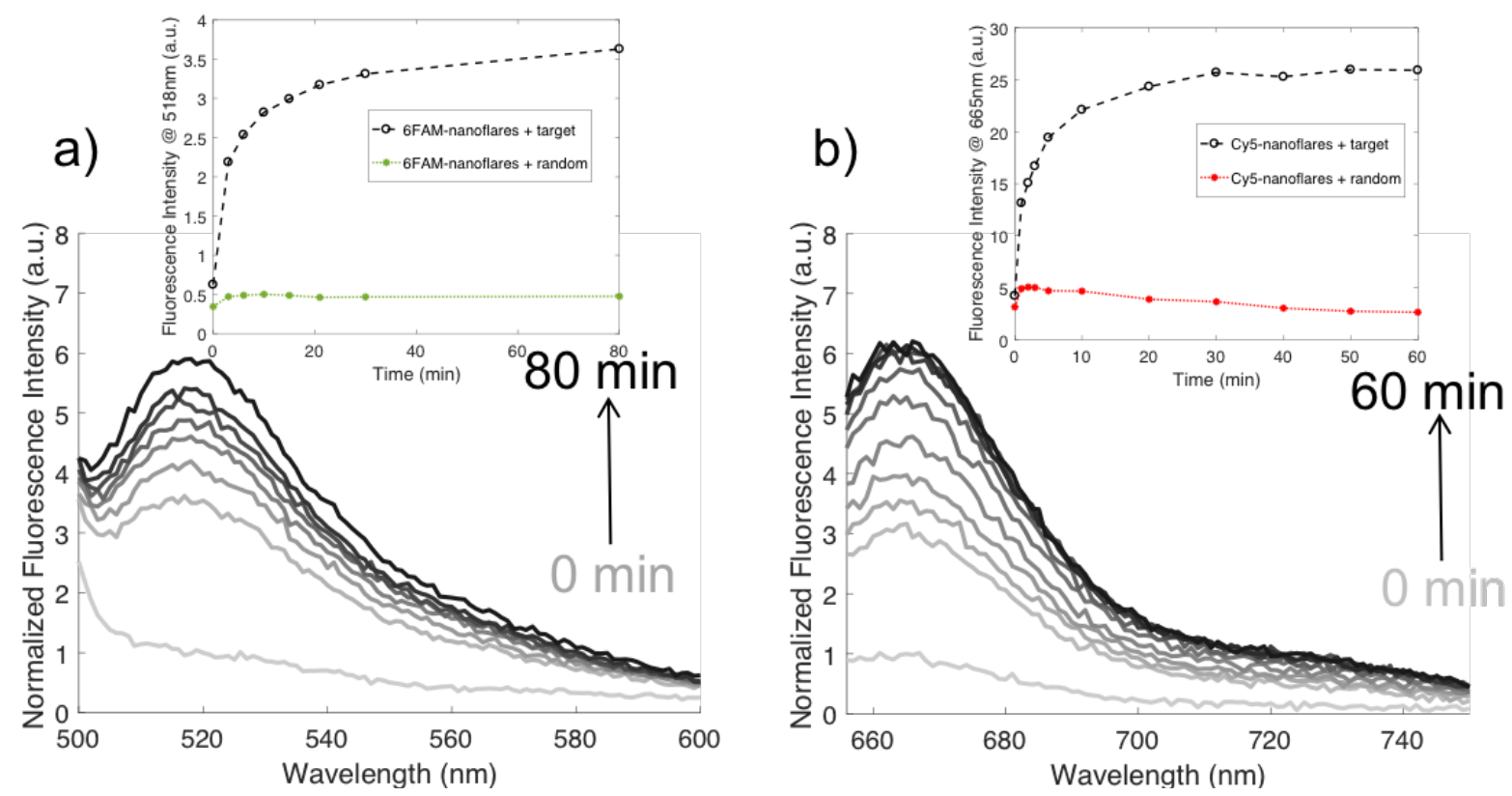

Figure S9: Fluorescence spectral changes and intensity profiles (insets) of nanoflares labeled with either a) 6-FAM or b) Cy5 in the presence of target/random oligo sequences.

a)

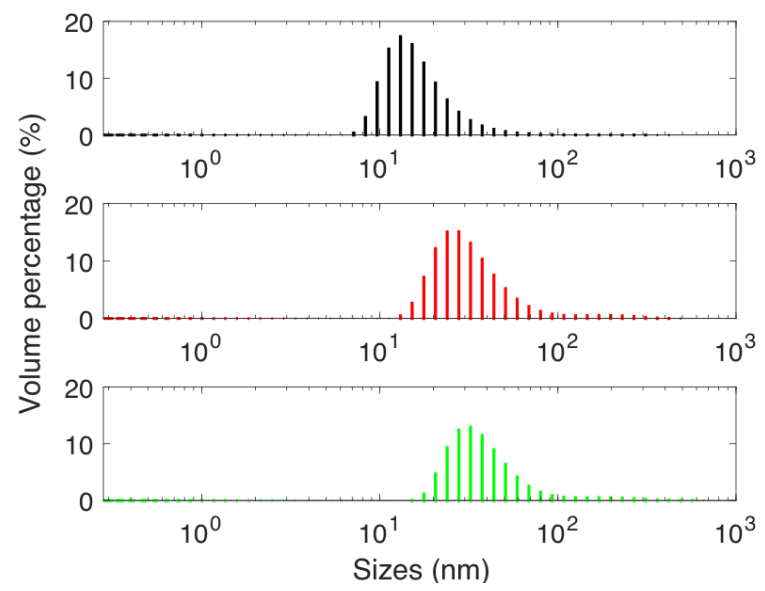

b)

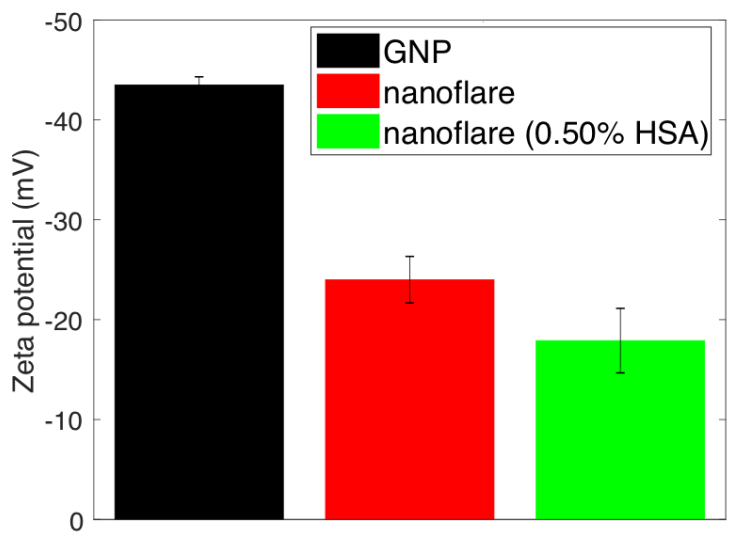

Figure S10: DLS measurements of the a) size and b) charge of the nanoflares before and after oligonucleotides functionalization as well as after protein corona formation. 
a)
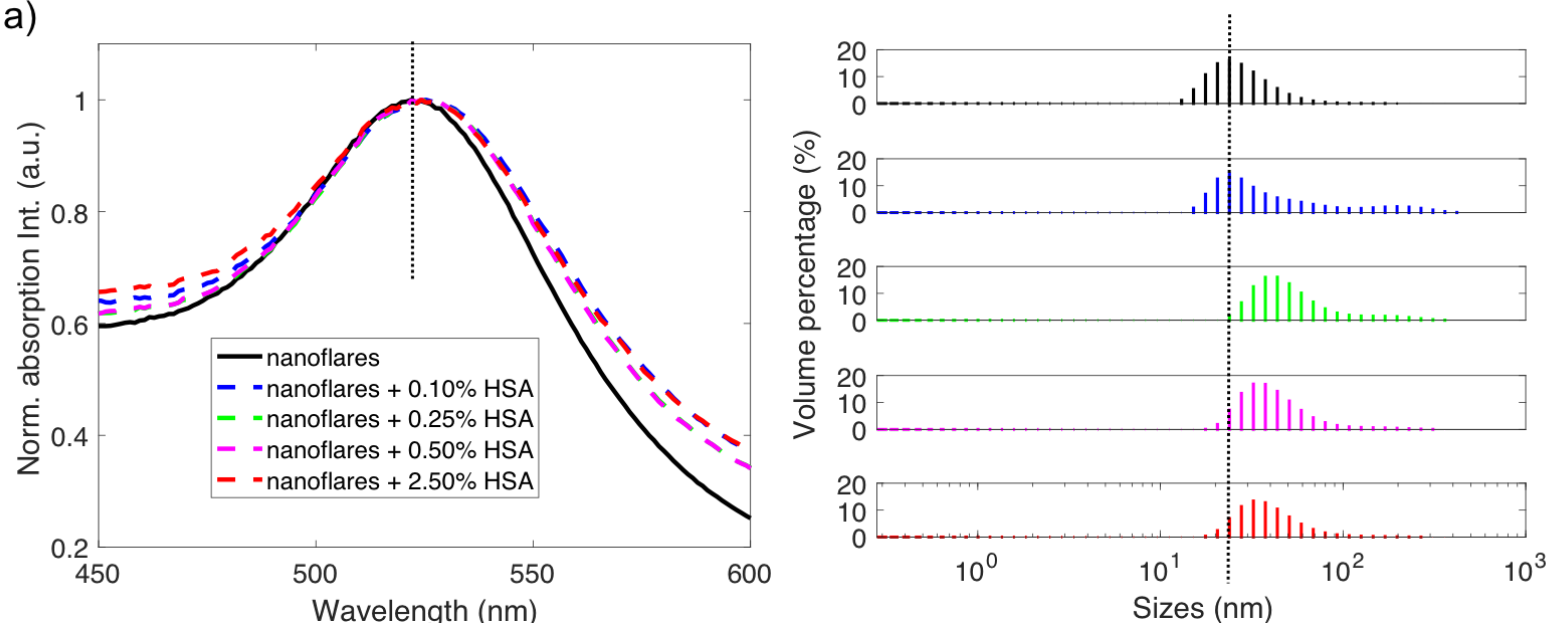

Figure S11: a) Optical extinction measurements and b) DLS size measurements of the nanoflares before and after protein incubation at varying concentrations of proteins.

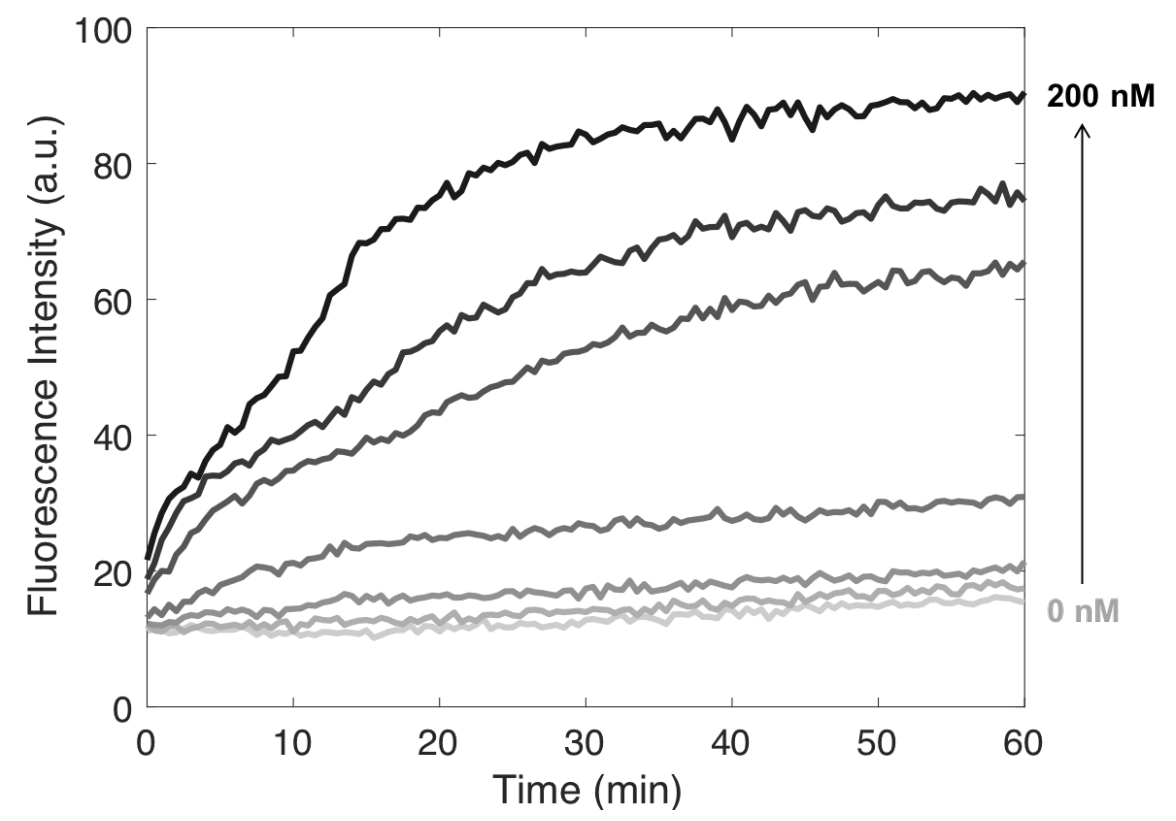

Figure S12: Fluorescence kinetics measured at $525 \mathrm{~nm}$ (485 nm excitation) for 6-FAM labelled nanoflares over 1 hour after the addition of an increasing concentration of complementary targets ( $0 \sim 200 \mathrm{nM}$ ). Based on the fluorescence calibration curve (Fig. S4), we can estimate that the percentage of released flares when the target concentration is increased to $200 \mathrm{nM}$ is 50\%. A rather low flare release rate might be associated with an increasing enthalpic penalty due to steric hindrance, electrostatic barrier upon more target binding. ${ }^{5}$ 
Table S1:

Nanoflare sequence design

\begin{tabular}{|l|l|}
\hline Sensing sequence & GTCCGACTACCAGACAGATTGATCCAATGT ${ }_{6}$-SH \\
\hline Flare sequence & $\begin{array}{l}\text { /5Cy5/TCATTGGATCAATCT } \\
\text { /5FAM/CATTGGATCAATCTGT }\end{array}$ \\
\hline Target sequence & CATTGGATCAATCTGTCTGGTAGTCGGAC \\
\hline 1-mismatched & CATTGGATCAATCAGTCTGGTAGTCGGAC \\
\hline 2-mismatched & CATTGGATGAATCTGTCTGCTAGTCGGAC \\
\hline Random sequence & GAGAGGGTAATCAGCCGTGTTCACCAACA \\
\hline
\end{tabular}

\section{References:}

1. Yuan, H. K., Khoury, C. G., Hwang, H., Wilson, C. M., Grant, G. A., Vo-Dinh, T. (2012) Nanotechnology 23, 075102.

2. Prigodich, A. E., Seferos, D. S., Massich, M. D., Giljohann, D. A., Lane, B. C., Mirkin, C. A. (2009) ACS Nano 3, 2147-2152.

3. Lytton-Jean, A. K. R., Mirkin, C. A. (2005) J. Am. Chem. Soc. 127, 12754-12755.

4. Chen, S. Y., Lazarides, A. A. (2009) J. Phys. Chem. C. 113, 12167-12175.

5. Randeria, P. S., Jones, M. R., Kohlstedt, K. L., Banga, R. J., Olivera de la Cruz, M., Schatz, G. C. , Mirkin, C. A. (2015) J. Am. Chem. Soc. 137, 3486-3489. 